\title{
Exploiting heterogeneous features to improve in silico prediction of peptide status - amyloidogenic or non-amyloidogenic
}

\author{
Smitha Sunil Kumaran Nair ${ }^{1 *}$, NV Subba Reddy ${ }^{2}$, KS Hareesha ${ }^{1}$ \\ From Asia Pacific Bioinformatics Network (APBioNet) Tenth International Conference on Bioinformatics - First \\ ISCB Asia Joint Conference 2011 (InCoB2011/ISCB-Asia 2011) \\ Kuala Lumpur, Malaysia. 30 November - 2 December 2011
}

\begin{abstract}
Background: Prediction of short stretches in protein sequences capable of forming amyloid-like fibrils is important in understanding the underlying cause of amyloid illnesses thereby aiding in the discovery of sequence-targeted antiaggregation pharmaceuticals. Due to the constraints of experimental molecular techniques in identifying such motif segments, it is highly desirable to develop computational methods to provide better and affordable in silico predictions.

Results: Accurate in silico prediction techniques of amyloidogenic peptide regions rely on the cooperation between informative features and classifier design. In this research article, we propose one such efficient fibril prediction implementation exploiting heterogeneous features based on bio-physio-chemical (BPC) properties, auto-correlation function of carefully selected amino acid indices and atomic composition within a protein fragment of amino acids in a window. In an attempt to get an optimal number of BPC features, an evolutionary Support Vector Machine (SVM) integrating a novel implementation of hybrid Genetic Algorithm termed Memetic Algorithm and SVM is utilized. Five prediction modules designed using Artificial Neural Network (ANN) models are trained with independent and integrated features in order to validate the fibril forming motifs. The results provide evidence that incorporating new feature namely auto-correlation function besides BPC, attempt to strengthen the sequence interaction effect in forming the feature vector thereby obtaining better prediction quality in terms of sensitivity, specificity, Mathews Correlation Coefficient and Area under the Receiver Operating Characteristics curve.

Conclusion: A significant improvement in performance is observed by introducing features like auto-correlation function that maintains sequence order effect, in addition to the conventional BPC properties selected through a novel optimization strategy to predict the peptide status - amyloidogenic or non-amyloidogenic. The proposed approach achieves acceptable results, comparable to most online predictors. Besides, it compensates the lacuna in existing amyloid fibril prediction tools by maintaining equilibrium between sensitivity and specificity.
\end{abstract}

\section{Background}

Amyloid-like fibrils may be formed from amylome, the universe of protiens. Today the association between protein fibrils and amyloid diseases, including Alzheimer's and prion diseases has been established [1]. To find a solution for effective treatments of amyloid disorders, the

\footnotetext{
* Correspondence: smitha.sunil@manipal.edu

'Department of Computer Science and Engineering, Manipal Institute of

Technology, Manipal University, Karnataka, India

Full list of author information is available at the end of the article
}

fundamental problem of understanding the factors that stimulate conformational changes and aggregation in proteins need to be solved [2].

The inference that there is a predisposition for primary sequence-specific formation of amyloidal fibrils is made from the wet lab proven experimental remarks that not all proteins are amyloidogenic and that only precise continuous stretches of amyloid fibril forming peptides are more amyloidogenic than other regions. Furthermore, the observation that amyloids can be formed from short

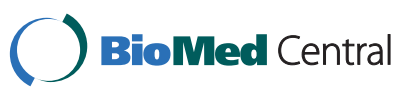

(c) 2011 Nair et al; licensee BioMed Central Ltd. This is an open access article distributed under the terms of the Creative Commons Attribution License (http://creativecommons.org/licenses/by/2.0), which permits unrestricted use, distribution, and reproduction in any medium, provided the original work is properly cited. 
peptide fragments, seem to indicate that these segments, which are exposed to the environment, can cause the changeover of native proteins into amyloid state [2].

It is apparent that certain sequences have more amyloidogenity than others regardless of studies that seem to suggest that assembly into amyloid-like fibrils is an intrinsic property of peptides, irrespective of their sequence. Additionally, some short segment of peptides have same amyloid characteristics as full length proteins, and some fragments have been considered to be the regions causing aggregation, due to the fact that they can transform the amyloidogenic tendencies of polypeptides by favouring or obstructing the formation of fibrils. These data recommend that primary sequence can impact the formation of amyloid fibrils, and has stimulated the recent advancement of computational models to predict the amyloidogenic propensities of proteins [3].

The challenge of predicting amyloidogenic regions has resulted in a variety of multi-parametric methods that attempt to predict such motif sequences [4]. Each methodology has its own hypothesis and implements, ranging from simple to complex [5]. Overall, the success of different computational approaches in predicting aggregationprone regions allow proposing that aggregation propensity in polypeptide chains is ultimately dictated by the sequence [6]. As research continues for the understanding of amyloid formation, the development of computational prediction techniques is an imperative supplement to experimental molecular approaches [5]. Several computational tools for predicting amyloid segments have emerged since 2004, such as [3,5-8] based on physicochemical grounds or structural denominators. However, methods by means of supervised machine learning models are only few such as Pafig [9].

In this article, we propose a supervised machine learning architecture that purely follows a sequence-based design strategy to determine the amyloidogenic short stretches in peptides. The systematically selected BPC properties of amino acids taken from Amino Acid index database in DBGet (Japan) and ProtScale in Swiss Expasy are utilized along with auto-correlation function and atomic composition within a peptide fragment to represent protein sequence features, and finally ANN is implemented to classify the fibril forming and non-fibril forming peptide segments. Prior to prediction, a feature optimization scheme based on evolutionary SVM is employed to search for the significant BPC features thereby reducing the dimensionality of the input space. The evolutionary strategy hybridizes a variant of Genetic Algorithm (GA) named Memetic Algorithm (MA) with SVM. The present study was initiated in an attempt to improve the overall performance in predicting the amyloid motifs in proteins by incorporating auto-correlation functions generated from selected amino acid indices and atomic composition of amino acids combined with corresponding BPC features obtained by a novel implementation of feature optimization.

\section{Results and discussion}

Given the laborious nature of experimental validation of peptide segments most prone to form fibrils, it is imperative that computational approaches be developed that could produce reliable, affordable and testable in silico predictions [4]. By incorporating correlation of carefully selected amino acid indices through embedded SVM, among the residues within a window, we attempted to strengthen the sequence interaction effect in forming the feature vector thereby reducing the misclassifications. In fact, experimentally predicted amyloidogenic regions reported in different works do vary [10]. One possibility could be due to the fact that the sequences are examined under diverse conditions. Hence reliable identification of amyloid fibril stretches is challenging and difficult.

The cross validation and independent tests carried out on all five Prediction Models (PMs) showed that inclusion of feature like auto-correlation function significantly improved the sensitivity and specificity. Results of comparative studies on prediction models based on Sensitivity $\left(S_{n}\right)$ and Specificity $\left(S_{p}\right)$ are shown in Table 1 . As seen, maximum performance is obtained by $\mathrm{PM}_{3}$ that integrates newly introduced correlation feature and the least by $\mathrm{PM}_{2}$ trained with constituent atoms alone. The performance of $\mathrm{PM}_{3}$ is further analysed using Receiver operating Characteristic (ROC) curves in figure $1 . \mathrm{PM}_{3}$ tested with $\mathrm{BPC}$ features and autocorrelation function values gives an Area Under Curve (AUC) of .854. The effect of other models with integrated and independent features have been investigated and found that the AUCs got significantly decreased as can be interpreted from figure 1. From these evaluations, we could establish that BPC features along with autocorrelation function are sufficient in determining the peptide status - amyloigogenic or nonamyloidogenic computationanlly.

\section{Comparison with existing approaches}

The performance of the best PM is compared with two recently published prediction tools, FoldAmyloid [8] and

Table 1 Performance of five prediction models in terms of Sensitivity $\left(S_{n}\right)$ and Specificity $\left(S_{p}\right)$

\begin{tabular}{ccccc}
\hline & \multicolumn{2}{c}{ Cross validation test } & \multicolumn{2}{c}{ Independent test } \\
\hline Prediction models & $\mathrm{S}_{\mathrm{n}}$ & $\mathrm{S}_{\mathrm{p}}$ & $\mathrm{S}_{\mathrm{n}}$ & $\mathrm{S}_{\mathrm{p}}$ \\
$\mathrm{PM}_{1}$ & 74.4 & 72.8 & 70.7 & 69.3 \\
$\mathrm{PM}_{2}$ & 61.6 & 59.3 & 55.4 & 52.3 \\
$\mathrm{PM}_{3}$ & 82.3 & 80.2 & 77.8 & 80.1 \\
$\mathrm{PM}_{4}$ & 75.5 & 74.6 & 72.6 & 71.9 \\
$\mathrm{PM}_{5}$ & 78.2 & 75.4 & 76.4 & 74.7 \\
\hline
\end{tabular}




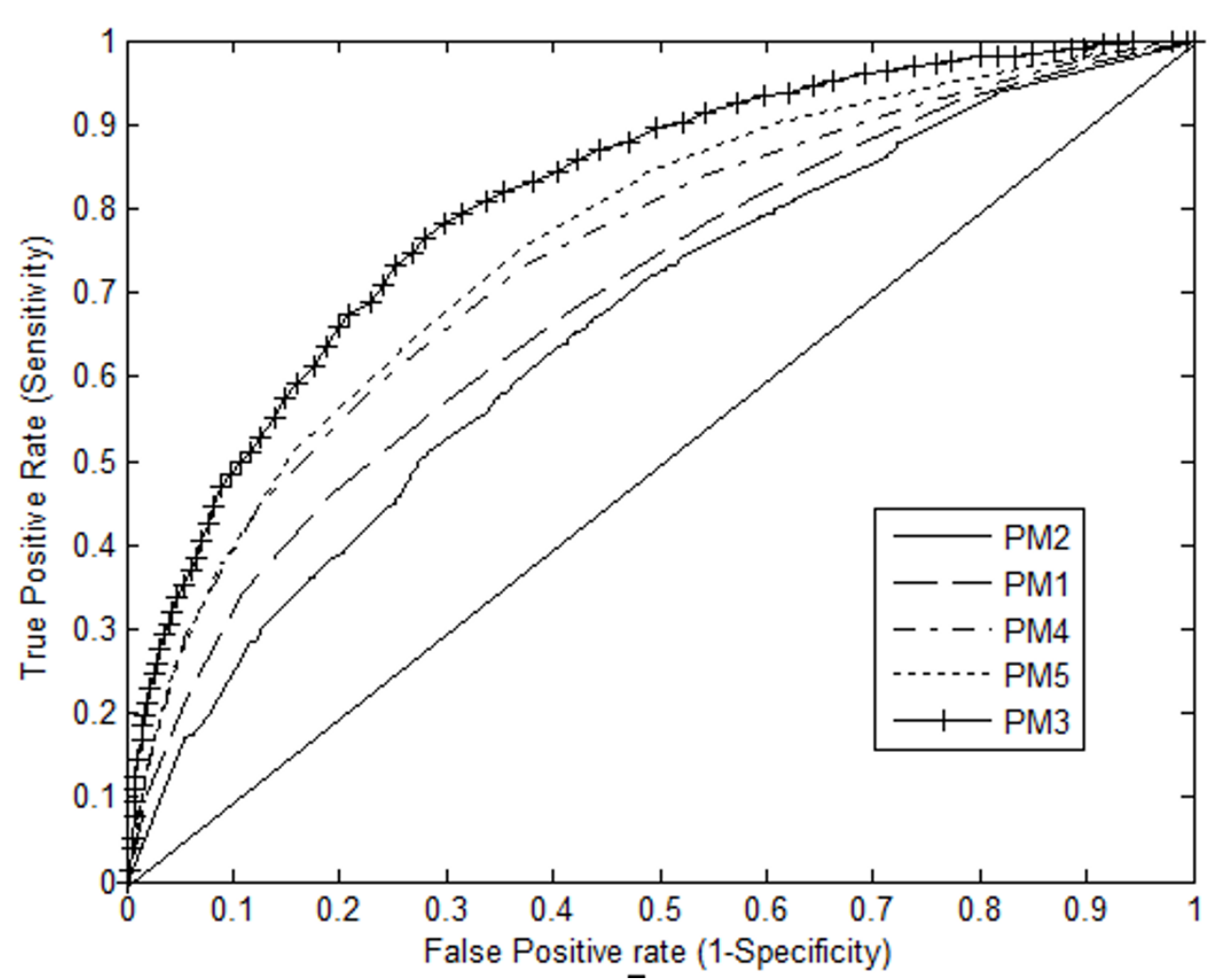

Figure 1 ROC plot of the true positive rate versus the false positive rate of five prediction models trained with independent and integrated features. The plot consists of five ROC curves on cross validation test (i) PM $_{1}$ trained with 40 features (BPC properties) (ii) PM $M_{2}$ trained with 5 features (atomic compositions within a residue) (iii) $\mathrm{PM}_{3}$ trained with 65 features (40 BPC with their 25 autocorrelation function values) (iv) $\mathrm{PM}_{4}$ trained with 45 features (40 BPC with 5 atomic values) and (v) 70 features (40 BPC properties, 25 autocorrelation function values and 5 atomic compositions).

AMYLPRED [5] which predict amyloidogenic regions from primary sequence, in terms of $S_{n}, S_{p}$, Balanced Accuracy (BACC) [11] and Mathew's Correlation Coeffecient (MCC). Prediction by FoldAmyloid is based on expected probability of hydrogen bond formation and expected packing density of residues and we chose the value of sliding window size and reliable frame size to be 6 to carry out the analysis. The tool AMYLPRED makes a consensus prediction of fibril forming regions in proteins by utilizing five diverse and individually published methods and the analysis was performed using the default parameters for each employed algorithm.

In order to compare various methods, their performances are evaluated on the same dataset. As the online predictors included in our analysis were not assigned any prediction cutoff or threshold, ROC curves cannot be constructed completely for these tools. Therefore, their performances in terms of $S_{n}$ and $S_{p}$ are denoted by specific points on the ROC plot. Figure 2 shows the scatter plot for true positive rate $\left(S_{n}\right)$ versus false positive rate $\left(1-S_{p}\right)$ to compare the balance between $S_{n}$ and $S_{p}$ of the proposed method with other online tools. The plot area is split into four quadrants denoted I-IV as referred [12]. In fact, the four quadrants denote algorithm that achieves (i) higher $S_{n}$ but lower $S_{p}$ (ii) higher $S_{n}$ and higher $S_{p}$ (iii) lower $S_{n}$ but higher $S_{p}$ (iv) lower $S_{n}$ and lower $S_{p}$. The diagonal line $(0,0)-(1,1)$ indicates a method that results in equal true positive rates and false positive rates. Hence, methods in quadrant II, far-off from the diagonal line are better performers.

Those methods that have its place in quadrant III have a tendency to predict all the examples as negative resulting in high specificity but very low sensitivity. FoldAmyloid and AMYLPRED appearing in quadrant III imply that although they have good specificity, (scores of .89 and .87 respectively), the sensitivity (scores of .32 and .26 respectively), is very poor. Out of these tools, AMYLPRED achieves the least BACC. MCC scores obtained by FoldAmyloid and AMYLPRED are .36 and .27 respectively.

Quadrant II in the plot is the best with both $S_{n}$ and $S_{p}$ being $>0.5$. As evident from the plot, the best proposed prediction model is found in this quadrant. Our method shows the highest sensitivity with the optimum specificity than previously reported prediction tools. Remarkably, 


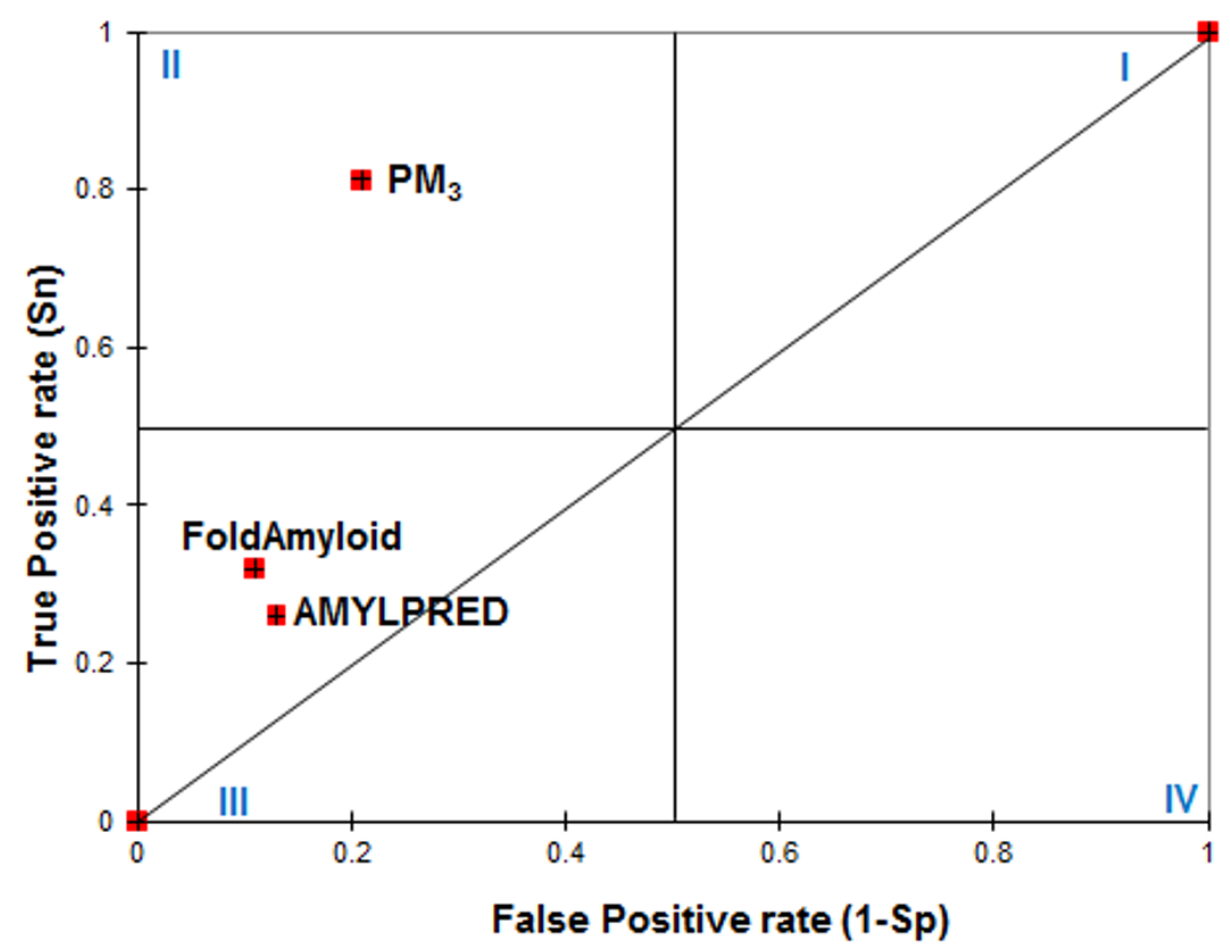

Figure 2 Scatter plot of the true positive rates (sensitivity) versus the false positive rates (1-specificity) for various fibril prediction methods. The plot consists of three points to illustrate that the proposed prediction model $\mathrm{PM}_{3}$ is superior in maintaining balance between true positive and false positive rates compared to other existing online tools.

the prediction results of our method are moderate and it achieves $S_{n}$ and $S_{p}$ of $82.3 \%$ and $80.2 \%$ for cross validation test and $77.8 \%$ and $80.1 \%$ for independent test. The presented computational architecture $\left(\mathrm{PM}_{3}\right)$ achieves the best BACC on an average of $80 \%$ and MCC score of .59 . Although [8] and [5] show high specificity, the overall balanced accuracy is poor due to very low sensitivity. Nevertheless, the sensitivity of these tools decreased substantially for Amylhexset data or they suffered from highly biased prediction (very low sensitivity but very high specificity). Low sensitivity obtained by FoldAmyloid may be due to the fact that trans-membrane and signal regions are not considered in their study. Frousios et al., [5] reported that scores of .13 and .95 representing sensitivity and specificity respectively are obtained by AMYLPRED for a dataset consisting of 5006 data samples used in their work. Garbuzynskiy et al. [8] correctly predicted $80 \%$ and $72 \%$ of amyloidogenic peptides and non-amyloidogenic peptides respectively for a dataset of 407 peptides using FoldAmyloid webserver.

The statistical measures, $S_{n}$ and $S_{p}$, the equilibrium maintained between them in terms of balanced prediction accuracy and MCC across the test dataset indicate that our algorithm produces the most significant improvement. The influence of each separate feature may be lesser; however the progress on the performance of the algorithm is further apparent when these features were taken into account collectively. Instead of randomly choosing some physicochemical properties related to aggregation for training the model, we tried to get an optimized set of properties related to fibrillar aggregates using a supervised machine learning algorithm named evolutionary SVM through a novel implementation of the memetic algorithm. As reviewed [13] and discussed [14], structural information of peptides plays substantial role in determining amyloid fibrils. In fact, the selected BPC features include few amino acid indices related to protein structure study described in PROFEAT [15] which indicates that the presented models are trained with a feature subset that contains structural details of amino acid sequence as well. Though the results of our prediction model matched favourably with other methods, it needs to be enhanced further. Improvement in prediction results may be possible by identifying novel relevant features, features that maintain the sequence interaction effect and by incorporating more training data.

\section{Conclusion}

The study of folding and unfolding events in proteins and subsequent aggregation into amyloid fibrillar deposits is becoming central to develop rational therapeutic strategies against devastations such as Alzheimer and Parkinson 
disease. A promising approach to spot such deposits is through computational prediction models. Even though, these models cannot replace the wet lab work, they might serve in identifying the regions of interest for further molecular investigations.

In our present study, due to the sheer amount of properties contained within the amino acids, we tried to provide a new and complementary set of physicochemical and biochemical properties through evolutionary SVM feature selection approach, besides their correlation and atomic composition within a residue in forming the feature vector to train the ANN model. To our knowledge, this is the first attempt to utilize auto-correlation function and atomic composition in computationally predicting the amyloidogenic or non-amyloidogenic peptide status. In addition, a variant and novel implementation of hybrid GA termed MA is implemented. Among the five prediction models built, $\mathrm{PM}_{3}$ - the one trained with 65 features gives the best results in terms of $S_{n}, S_{p}$ and AUC of ROC curve which clearly provide indication that newly introduced feature, autocorrelation function which helps in maintaining the sequence order effect, besides the BPC properties selected through MA have high impact in determining the amyloid aggregates. As also evident from the MCC score and the scatter plot, the proposed in silico computing method achieves an agreeable result and preserves balance between the rates of true positives and false positives that is deficit in the existing online tools.

\section{Methods}

\section{Sequence dataset gathering}

The accuracy of fibril motif prediction can be enhanced with the use of soft computing approaches. However, the classifiers are required to be trained with appropriate datasets in the form of positive and negative data. We compiled experimentally proved proteins related to amyloidosis and proteins with no experimentally determined amyloidogenic regions published in literature $[2,3,5-7,16,17]$, in order to construct the dataset (Additional file 1). The extracted sequences associated with protein aggregation include natively globular proteins, natively intrinsically unstructured proteins, amyloidogenic proteins and proteins related to depositional diseases. All protein sequences were downloaded in Fasta format from Uniprot-Swissprot database [18]. The wet lab analysis of different proteins reveals that these peptide sequences contain short stretches which form amyloid fibrils [8]. Thompson et al., [7] claim that hexpeptides are sufficient for forming amyloid-like fibrils. Therefore, a dataset of hexpeptides including positive and negative examples of fibril formation was prepared by sliding a window of six residues. We term this dataset Amylhexset. A dataset of 2512 hexpeptides of which 1232 that have been experimentally proved to form fibril forming segments and 1280 that have provided negative results in fibril forming assessment form Amylhexset.

\section{Feature encoding and mining}

The overall capability of machine learning models to identify fibril aggregates is built on the encoded features of the dataset. ANN model needs each instance of data to be denoted in the form of real vectors. Therefore, the numerals of physiochemical or biochemical properties of amino acids in addition to their auto-correlation functions and atomic composition within a protein fragment are utilized to form the feature vector.

A collection of bio-physio-chemical characteristics of amino acids are proved to be supportive in studying protein macroscopic properties like aggregation, performing comparison among sequences or understanding conservation of functionally significant fragments in a peptide family (physio-chemical signatures). As these properties are proved to be useful in studying protein sequence profiles, folding and function, we have taken them into consideration for fibril motif identification. Moreover, computational approaches based on physio-chemical grounds have shown relatively good performances in predicting aggregation propensity $[5,6,8,17]$. The Amino Acid index (AAindex Version 9) [19] provides 544 characteristics for each of the 20 amino acids. Among the 544 indices, 13 were never considered due to partial data. According to Mathura et al., [20] properties with insufficient data and least relevant indices with respect to the study of protein structure, function and sequence are omitted in their database named APDbase [21]. Therefore, among all 531 features in AAindex database, only 246 are taken into account in APDbase. Of the 246 entries in APDbase, the last 29 entries (except MAXF760101 Normalized frequency of alpha-helix with description index 226 in APDbase) correspond to ProtScale in Swiss Expasy [22] which are not endowed with IDs or Accession Nos and the remaining 216 properties are from AAindex database. The authors have designated certain Accession Nos in a similar fashion as those of in AAindex version 9 for the very last 28 properties available in APDbase. Thus 531 in [19] +28 in [22] indices were evaluated for potential use.

Experiments carried out by Goldscmidt et al., [1] suggest that if a fibrillizing sequence fragment is shuffled, the reordered segment loses its ability to form fibril aggregates. Keeping this in mind, a new feature namely auto-correlation function is introduced. Li et al., [15] suggest that auto-correlation feature describe the level of correlation between the amino acids in terms of their selected physico-chemical property within a residue. Moreau-Broto auto-correlation function of amino acid indices inspired from the work of [23] on pinpointing disordered regions in proteins is utilized. According to Han et al., [23] 
prediction accuracy may be improved by incorporating this function as it could test if the BPC property of an amino acid is dependent of that of its neighbours and has been used in the protein structural and functional classification studies. However, this was not effective in the prediction of membrane proteins [24]. The Moreau-Broto auto-correlation function $F_{v}$ of an amino acid index is calculated within a window, as:

$$
F_{v}=\frac{1}{w-v} \sum_{i=1}^{w-v} p_{i}^{*} p_{i+v}(v=1,2,3,---, w-1)
$$

where $w$ is the window size, $p_{i}$ and $p_{i+v}$ are the amino acid index values at positions $i$ and $i+v$ respectively [23]. Here, $w=6$ and hence $F_{v}(v=1,2,--, 5)$ for the best 5 BPC properties selected through feature pre-optimization (discussed in the subsequent section) is calculated.

Atomic composition (AC) refers to Carbon, Hydrogen, Nitrogen, Oxygen and Sulphur atoms in an amino acid sequence. As the count of constituent atoms in each hexmer varies from one another, this feature is hypothesised to be a good choice as it helps in differentiating samples. Therefore, atomic values of samples in Amylhexset are included as five features in the encoding scheme. However, this feature does not contribute in maintaining the sequence order effect due to the fact that $\mathrm{AC}$ (hexmer) = AC (shuffled hexmer).

The values of each property were scaled so as to fall within a small specified range using min-max normalization technique [25]. Formulation of min-max normalization:

$$
v^{\prime}=\frac{v-\min _{P}}{\max _{P}-\min _{P}}\left(n e w_{-} \max _{P}-n e w_{-} \min _{P}\right)+n e w_{-} \min _{P}
$$

Suppose that $\min _{P}$ and $\max _{P}$ are the minimum and maximum values of a feature, $P$, then min-max normalization maps a value, $v$, of $P$ to $v^{\prime}$ in the range $\left[n e w \_\min _{P}\right.$, $\left.n e w \_\max _{P}\right]$ by computing equation (2). This transformation prevents features in greater numeric ranges from dominating those in smaller numeric ranges.

\section{Feature optimization}

In order to attain a considerably improved performance in terms of classification ability, it is a prerequisite to generate relevant features so as to discriminate well among classes. One of the basic problems in computational biology is how effectively a lesser subset of significant features be selected [10]. Feature optimization involves two essential tasks: (i) feature pre-optimization [26]; and (ii) determining the best subset of features from pre-optimized set of features. The latter is achieved through evolutionary SVM, a method that is inspired from the work by Huang et al., [27] on protein subnuclear localization.
Filter based and embedded based models [28] were employed and evaluated for pre-optimization. In this regard, embedded model based on SVM classifier is found to be more effective in selecting an initial round of BPC properties and 186 properties are selected. The ultimate set of BPC properties are selected by evolutionary SVM utilizing a variant of hybrid GA termed MA. It is believed that one of the important factors affecting the GA results is due to the varying implementation of the GA method [10]. In this study, one such variation of hybrid GA resulting in MA is adopted.

The proposed MA concisely presented in figure 3 showing the representation of feature vector of intermediate steps in the optimization procedure selects a subset out of 186 pre-selected features, and determines the corresponding SVM parameter values using 5-fold cross-validation as an estimator of generalization ability. For this purpose, LIBSVM [29] is trained with Amylhexset using Radial Basis Function (RBF) kernel.

MA is an evolutionary strategy where elements of local optimization are incorporated in a conventional GA global search to increase the precision of the solution [30]. Eiben and Smith [31] suggest that local search acting on solutions created by mutation or recombination result in better solutions. Zhu et al., [32] propose a wrapper-filter dimensionality reduction methodology using a memetic outline. Predominantly, the approach adds or removes a feature from a feature subgroup based on their ranking. It has been shown in the literature that MA as an optimization technique utilizing filter ranking methods, result in the enhancement of classification performance. The pseudo code for MA is shown in figure 4. The major steps involved in MA are briefly discussed:

Initialization: Create an initial population with a set of randomly generated 10 parents with 40 properties each. This initial creation permits the GA to discover all possible range of solutions which support the most likely results to a great extent.

Evaluation: Compute fitness values of all individuals using SVM with 5-fold cross validation in the population and they are ranked according to their fitness.

Selection: With this approach, greater the fitness, higher the chance to move ahead to subsequent generation. To ensure that good individuals do survive to next generations, we choose the best half according to the fitness.

Recombination: A property pool array is defined to hold the shuffled properties of selected best parents. For every offspring, the properties in the pool were drawn one by one, saved if the property occurs the first time for the specific offspring else put back in to the pool. With this procedure it could be made sure, that a property which appeared more often in the fitter parents has a higher probability to be a part of the new generation. 

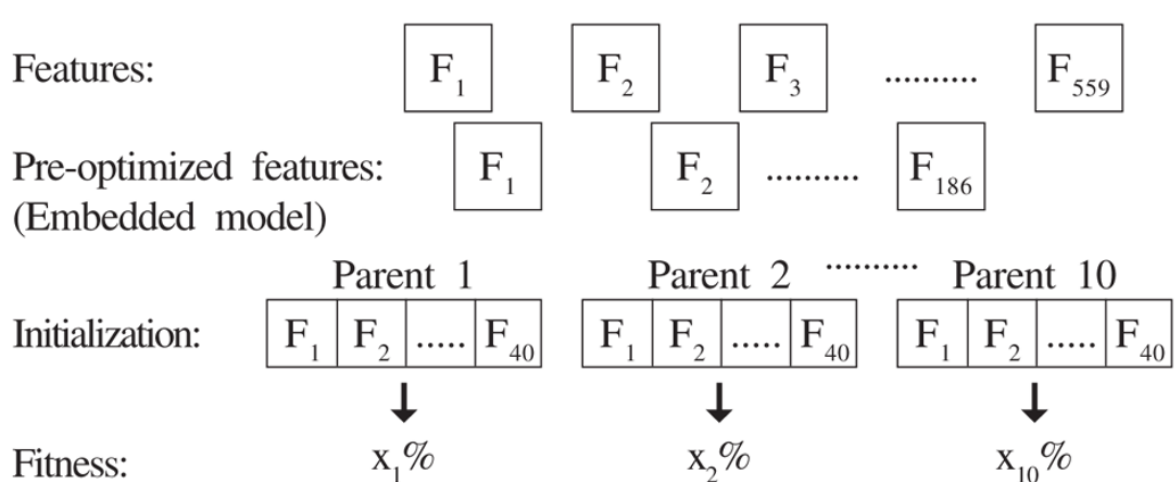

Parent 1

Parent 2 Parent 5

Selection:

\begin{tabular}{|l|l|l|l|}
\hline $\mathrm{F}_{1}$ & $\mathrm{~F}_{2}$ & $\ldots .$. & $\mathrm{F}_{40}$ \\
\hline
\end{tabular}

\begin{tabular}{|l|l|l|l|}
$\mathrm{F}_{1}$ & $\mathrm{~F}_{2}$ & $\ldots .$. & $\mathrm{F}_{40}$ \\
\hline
\end{tabular}

\begin{tabular}{|l|l|l|l|}
\hline $\mathrm{F}_{1}$ & $\mathrm{~F}_{2}$ & $\ldots .$. & $\mathrm{F}_{40}$ \\
\hline
\end{tabular}

Recombination: Offspring 1

Offspring $2 \cdots \cdots$ Offspring 5

\begin{tabular}{|c|c|c|c|c|c|c|c|c|c|}
\hline \multicolumn{2}{|c|}{ Mutation: } & \multicolumn{2}{|c|}{$\mathrm{F}_{1}$} & $\mathrm{~F}_{2}$ & $\ldots .$. & $\mathrm{F}_{40}$ & $\mathrm{~F}_{1}$ & \multicolumn{2}{|c|}{\begin{tabular}{|l|l|}
$\mathrm{F}_{2}$ & $\ldots .$. \\
\end{tabular}} \\
\hline & & $\mathrm{F}_{1}$ & $\mathrm{~F}_{2}$ & $\mathrm{~F}_{3}$ & $\mathrm{~F}_{4}$ & $\mathrm{~F}_{5}$ & $\mathrm{~F}_{6}$ & & $F_{186}$ \\
\hline \multirow{5}{*}{$\begin{array}{l}\text { Local } \\
\text { search: }\end{array}$} & $\mathrm{O}_{\mathrm{D}}$ & 1 & 0 & 1 & 0 & 0 & 0 & ..... & \\
\hline & $\mathrm{O}$ & 1 & 0 & 1 & 0 & 0 & 1 & $\ldots .$. & \\
\hline & $\mathrm{O}_{\mathrm{A}}$ & 1 & 0 & 1 & 0 & 1 & 1 & $\ldots$. & \\
\hline & & $\mathrm{F}_{1}$ & $\mathrm{~F}_{2}$ & $\mathrm{~F}_{3}$ & $\mathrm{~F}_{4}$ & $\mathrm{~F}_{5}$ & $\mathrm{~F}_{6}$ & & $F_{186}$ \\
\hline & $\mathrm{O}_{\mathrm{N}}$ & 1 & 0 & 1 & 0 & 1 & 0 & ..... & $\ldots .$. \\
\hline
\end{tabular}

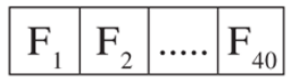

Feature Rank
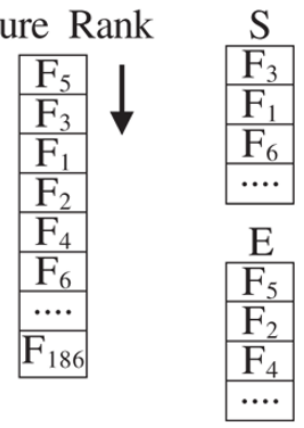

$\left.\begin{array}{lllll}\text { Improvement: } & \mathrm{O}_{\mathrm{N}_{1}} & \mathrm{O}_{\mathrm{N}_{2}} & \cdots & \mathrm{O}_{\mathrm{N}_{5}} \\ {\left[\text { If fitness }\left(\mathrm{O}_{\mathrm{N}}>\mathrm{O}\right)\right.}\end{array}\right]$

Figure 3 Overview of feature optimization strategy. This gives an overview of feature optimization strategy.

Mutation: Mutation makes sure that the properties, which are not part of the first generation, have a chance to get into the algorithm later. A high mutation rate is desired for the first few generations, because it allows making big steps towards a better accuracy, but it should decrease with every generation to allow the algorithm to find the optimum with small changes. To set the number of mutations per offspring, we implemented an exponential function [10], depending on the number of actual generation.

$$
N_{M}=s \cdot m \cdot e^{-6 \frac{n_{G}}{N_{G}}}
$$

where $\mathrm{N}_{M}$ is the number of mutations per offspring during the actual generation $\mathrm{n}_{\mathrm{G}}, \mathrm{s}$ is the size of offspring and $\mathrm{m}$ is the mutation value, a constant between 0 and
1 which defines the start value of the first generation depending on the size of an offspring. $\mathrm{N}_{\mathrm{G}}$ stands for the total number of generations the algorithm is going to run for. In this work, the mutation value has been set to a value of 0.2 for every test.

Improvement through local search: Maintain an array of binary string of length equivalent to pre-optimized features for each offspring, so that each bit encodes a single feature. A bit of ' 1 ' implies the corresponding feature is selected and a ' 0 ' that is excluded.

Given an Offspring O, we define two sets $\mathrm{S}$ and $\mathrm{E}$ for Selected and Excluded properties represented in offspring respectively. Filter ranking method [32], F-statistic [10] has been used for ranking $S$ and $E$ with the most vital feature ranked the maximum score.

Two basic local search operators suggested by Zhu et al., [32] are defined namely (i) Add: Select a high 
Begin

INITIALIZE population;

While (convergence criterion not reached)

EVALUATE the fitness of every individual;

SELECT parents;

RECOMBINE to produce offspring;

MUTATE offspring;

IMPROVE offspring via local search;

REPLACE individual with new version;

End while

End

Figure 4 Pseudo code for the memetic algorithm. The pseudo code for the memetic algorithm is shown.

ranked feature from $\mathrm{E}$ and add it to $\mathrm{O}$ resulting in $\mathrm{O}_{\mathrm{A}}$ and (ii) Delete: Select a low ranked feature from $S$ and delete it from $\mathrm{O}$ resulting in $\mathrm{O}_{\mathrm{D}}$.

The new offspring $\mathrm{O}_{\mathrm{N}}$ is formed by merging $\mathrm{O}_{\mathrm{A}}$ and $\mathrm{O}_{\mathrm{D}}$ such that the highly ranked features in $\mathrm{E}$ added to $\mathrm{O}_{\mathrm{A}}$ are retained and the low ranked features in $S$ excluded from $O_{D}$ are removed. Since prior information on the optimum number of properties is known, the number of bit ' 1 ' in each offspring is restricted to 40 . Local search length defining the maximum count of Add and Delete procedures in each local search is set to a value 8 .

For each mutated offspring $\mathrm{O}, \mathrm{O}_{\mathrm{N}}$ is created. Fitness functions for each pair of $\mathrm{O}$ and $\mathrm{O}_{\mathrm{N}}$ are evaluated using LIBSVM. If the classification ability in terms of cross validation rate of $\mathrm{O}_{\mathrm{N}}$ outperforms its corresponding $\mathrm{O}$, then the mutated offspring $\mathrm{O}$ is replaced by $\mathrm{O}_{\mathrm{N}}$. This improvement of offsprings through local search is continued for all mutated offsprings.

Replacement: A new population is formed by replacing the worst half discarded in selection process with the mutated improved offsprings.

Convergence criterion: The procedures of selection, recombination, mutation, local search and replacement continue till the convergence criterion is met which has been set to a maximum size of 100 generations.

The best combination of properties with cross validation rate of $83.34 \%$ was obtained in $89^{\text {th }}$ generation after which the accuracy remained constant. Therefore, 40 features acquired (Additional file 2) are utilized for feature vector representation.

\section{Building models on training data}

In this contribution, five prediction models $\left(\mathrm{PM}_{1}-\mathrm{PM}_{5}\right)$ based on ANN are trained and built with the state-ofthe-art implementation in Neural Network Pattern Recognition Tool of MATLAB R2008b that uses a twolayer feed-forward network with sigmoid output neurons. The PMs are trained with independent and integrated features such as (i) $\mathrm{PM}_{1}$ trained with 40 features (BPC properties) (ii) $\mathrm{PM}_{2}$ trained with 5 features (atomic compositions within a residue) (iii) $\mathrm{PM}_{3}$ trained with 65 features (40 BPC with their 25 autocorrelation function values) (iv) $\mathrm{PM}_{4}$ trained with 45 features (40 BPC with 5 atomic values) and (v) 70 features (40 BPC properties, 25 autocorrelation function values and 5 atomic compositions). Each PM has input nodes ranging from 5 to 70 depending on the dimension of the feature vector. The output layer of the model contains one unit with a target value ' 1 ' if motif is positive or ' 0 ' if motif is negative. The number of hidden layer units was selected as 23 , by trial and error method. The network is trained with back propagation algorithm utilizing sigmoid transfer function as the activation function. The training data is partitioned into three subgroups. $60 \%$ of the total data were utilized 
to train the ANN. 20\% each were used for validation and testing. In order to evaluate the performance of unobserved data that were not included in the training process, the model was further assessed by presenting another data subset comprising 1900 hexmer samples whose results are shown in Table 1.

\section{Additional material}

Additional file 1: Amylhexset This file contains the Genbank / Swissprot Accession Nos. of positive and negative data samples collected from the literature, which have been used for training and testing.

Additional file 2: AAindex Ids or Accession Nos. of 40 BPC properties used. This file shows the BPC properties selected by the memetic algorithm.

\section{Acknowledgements}

This article has been published as part of BMC Bioinformatics Volume 12 Supplement 13, 2011: Tenth International Conference on Bioinformatics First ISCB Asia Joint Conference 2011 (InCoB/ISCB-Asia 2011): Bioinformatics. The full contents of the supplement are available online at http://www. biomedcentral.com/1471-2105/12?issue=S13.

\section{Author details}

'Department of Computer Science and Engineering, Manipal Institute of Technology, Manipal University, Karnataka, India. ${ }^{2}$ Mody Institute of Technology and Science University, Rajasthan, India.

\section{Authors' contributions}

SSKN conceived the research, designed the code, and performed computational prediction, comparisons and statistical analysis. NVSR and HKS supervised the work and helped in manuscript preparation. All authors read and approved the final manuscript.

\section{Competing interests}

The authors declare that they have no competing interests.

Published: 30 November 2011

\section{References}

1. Goldschmidt L, Teng PK, Riek R, Eisenberg D: Identifying the amylome, proteins capable of forming amyloid-like fibrils. PNAS 2010, 107(No. 8):3487-3492.

2. Galzitskaya OV, Garbuzynskiy SO, Lobanov MY: Prediction of amyloidigenic and disordered regions in protein chains. PLOS Computational Biology 2006, 2(12):e177

3. Zhang Z, Chen $\mathrm{H}$, Lai L: Identification of amyloid fibril-forming segments based on structure and residue-based statistical potential. Bioinformatics 2007, 23(No. 17):2218-2225.

4. Nair SSK, Reddy NVS, Hareesha KS: Assessing the accuracy of computational tools for the prediction of amyloid fibril forming motifs: an overview. IJCA Special Issue on "Computational Science - New Dimensions \& Perspectives" NCCSE; 2011, 155-157.

5. Frousios KK, Iconomidou VA, Karletidi CM, Hamodrakas SJ: Amyloidogenic deteminants are usually not buried. BMC Structural Biology 2009, 9:44.

6. Conchillo-Solé O, de Groot NS, Avilés FX, Vendrell J, Daura X, Ventura S: AGGRESCAN: a server for the prediction of "hot spots" of aggregation in polypeptides. BMC Bioinformatics 2007, 8:65.

7. Thompson MJ, Sievers SA, Karanicolas J, Ivanova MI, Baker D, Eisenberg D: The 3D profile method for identifying fibril-forming segments of proteins. PNAS 2006, 103(No. 11):4074-4078.

8. Garbuzynskiy SO, Lobanov MY, Galzitskaya OV: FoldAmyloid: a method of prediction of amyloidogenic regions from protein sequence. Bioinformatics 2010, 26(No.3):326-332

9. The Pafig Server (Version 1.0). [http://www.mobioinfor.cn/pafig/]
10. Nair SSK, Reddy NVS, Hareesha KS: An evaluation of feature selection approaches in finding amyloidogenic regions in protein sequences. International Journal of Computer Applications 2010, 8(No.2):1-6.

11. Levner I: Feature selection and nearest centroid classification for protein mass spectrometry. BMC Bioinformatics 2005, 6:68.

12. Bandyopadhyay $S$, Mitra R: TargetMiner: microRNA target prediction with systematic identification of tissue-specific negative example. Bioinformatics 2009, 25(No. 20):2625-2631.

13. Caflisch $\mathrm{A}$ : Computational models for the prediction of polypeptide aggregation propensity. Current Opinion in Chemical Biology 2006, 10:437-444.

14. Marshall KE, Serpell LC: Insights into the structure of amyloid fibrils. The Open Biology Journal 2009, 2:185-192.

15. Li ZR, Lin HH, Han LY, Jiang L, Chen $X$, Chen YZ: PROFEAT: a web server for computing structural and physicochemical features of proteins and peptides from amino acid sequence. Nucleic Acids Research 2006, 34(Web Server issue):W32-W37.

16. López de la Paz M, Serrano L: Sequence determinants of amyloid fibril formation. PNAS 2004, 101(No. 1):87-92.

17. Tian J, Wu N, Guo J, Fan Y: Prediction of amyloid fibril-forming segments based on a support vector machine. BMC Bioinformatics 2009, 10(Suppl 1):S45.

18. UniProt Download Center. [http://www.ebi.ac.uk/uniprot/database/ download.html].

19. Kawashima S, Kanehisa M: AAindex: amino acid index database. Nucleic Acids Res 2008, 28(1):374.

20. Mathura VS, Kolippakkam D: APDbase: Amino acid Physicochemical properties Database. Bioinformation 2005, 1(1):2-4.

21. Amino acid Physical-chemical property Database. [http://www.rfdn.org/ bioinfo/APDbase.php].

22. ProtScale. [http://www.expasy.org/tools/protscale.html].

23. Han $P$, Zhang $X$, Feng ZP: Predicting disordered regions in proteins using profiles of amino acids indices. BMC Bioinformatics 2009, 10(Suppl 1):S42.

24. Cui J, Liu Q, Puett D, Xu Y: Computational prediction of human proteins that can be secreted into the bloodstream. Systems Biology 2008, 24(No. 20):2370-2375

25. Han J, Kamber M: Data mining - concepts and techniques. Elsevier, II 2008.

26. Peng $Y, W u Z$, Jiang J: A novel feature selection approach for biomedical data classification. Journal of Biomedical Informatics 2010, 43:15-23.

27. Huang WL, Tung CW, Huang HL, Hwang SF, Ho SY: ProLoc: Prediction of protein subnuclear localization using SVM with automatic selection from physicochemical composition features. BioSystems 2007, 90:573-581.

28. Saeys $Y$, Inza I, Larrañaga P: A review of feature selection techniques in bioinformatics. Bioinformatics 2007, 23(No. 19):2507-2517.

29. LIBSVM - A Library for Support Vector Machines. [http://www.csie.ntu. edu.tw/ cjlin/libsvm/].

30. Moscato P: Memetic algorithms: a short introduction; new ideas in optimization. McGraw-Hill Ltd. UK 1999.

31. Eiben $A E$, Smith JE: Introduction to evolutionary computing. Natural Computing Series Springer, Germany; 2003

32. Zhu Z, Ong YS, Dash M: Wrapper-Filter feature selection algorithm using a memetic framework. IEEE Transactions on Systems, Man, and Cybernetics Part B: Cybernetics 2007, 37(No. 1):70-76.

doi:10.1186/1471-2105-12-S13-S21

Cite this article as: Nair et al: Exploiting heterogeneous features to improve in silico prediction of peptide status - amyloidogenic or nonamyloidogenic. BMC Bioinformatics 2011 12(Suppl 13):S21. 